\title{
Exebacase for patients with Staphylococcus aureus bloodstream infection and endocarditis
}

\author{
Vance G. Fowler Jr., ${ }^{1,2}$ Anita F. Das, ${ }^{3}$ Joy Lipka-Diamond, ${ }^{4}$ Raymond Schuch, ${ }^{5}$ Roger Pomerantz, ${ }^{5}$ Luis Jáuregui-Peredo, ${ }^{6}$ \\ Adam Bressler, ${ }^{7}$ David Evans, ${ }^{8}$ Gregory J. Moran, ${ }^{9}$ Mark E. Rupp, ${ }^{10}$ Robert Wise, ${ }^{11}$ C. Ralph Corey, ${ }^{1}$ Marcus Zervos, ${ }^{12}$ \\ Pamela S. Douglas, ${ }^{1,2}$ and Cara Cassino ${ }^{5}$ \\ 'Duke University Medical Center, Durham, North Carolina, USA. ${ }^{2}$ Duke Clinical Research Institute, Durham, North Carolina, USA. ${ }^{3}$ AD Stat Consulting, Guerneville, California, USA. ${ }^{4}$ Lipka Consulting, Mullica \\ Hill, New Jersey, USA. ${ }^{5}$ ContraFect Corporation, Yonkers, New York, USA. ${ }^{5}$ Mercy Health-St. Vincent Medical Center, Toledo, Ohio, USA. Infectious Disease Specialists of Atlanta, Georgia, USA. ${ }^{8}$ The Ohio \\ State University, Columbus, Ohio, USA. ${ }^{9}$ Olive View-UCLA Medical Center, Sylmar, California, USA. ${ }^{0}$ University of Nebraska Medical Center, Omaha, Nebraska, USA. "Johns Hopkins Bayview Medical Center, \\ Baltimore, Maryland, USA. ${ }^{12 H e n r y ~ F o r d ~ H e a l t h ~ S y s t e m, ~ D e t r o i t, ~ M i c h i g a n, ~ U S A . ~}$
}

\begin{abstract}
BACKCROUND. Novel therapeutic approaches are critically needed for Staphylococcus aureus bloodstream infections (BSIs), particularly for methicillin-resistant S. aureus (MRSA). Exebacase, a first-in-class antistaphylococcal lysin, is a direct lytic agent that is rapidly bacteriolytic, eradicates biofilms, and synergizes with antibiotics.
\end{abstract}

METHODS. In this superiority-design study, we randomly assigned 121 patients with S. aureus BSI/endocarditis to receive a single dose of exebacase or placebo. All patients received standard-of-care antibiotics. The primary efficacy endpoint was clinical outcome (responder rate) on day 14.

RESULTS. Clinical responder rates on day 14 were $70.4 \%$ and $\mathbf{6 0 . 0 \%}$ in the exebacase + antibiotics and antibiotics-alone groups, respectively (difference $=10.4,90 \% \mathrm{Cl}[-6.3,27.2], P=0.31$ ), and were 42.8 percentage points higher in the prespecified exploratory MRSA subgroup ( $74.1 \%$ vs. $31.3 \%$, difference $=42.8,90 \% \mathrm{Cl}[14.3,71.4]$, ad hoc $P=0.01)$. Rates of adverse events (AEs) were similar in both groups. No AEs of hypersensitivity to exebacase were reported. Thirty-day all-cause mortality rates were $\mathbf{9 . 7 \%}$ and $\mathbf{1 2 . 8 \%}$ in the exebacase + antibiotics and antibiotics-alone groups, respectively, with a notable difference in MRSA patients (3.7\% vs. $25.0 \%$, difference $=-21.3,90 \% \mathrm{Cl}[-45.1,2.5]$, ad hoc $P=0.06$ ). Among MRSA patients in the United States, median length of stay was 4 days shorter and 30 -day hospital readmission rates were $48 \%$ lower in the exebacase-treated group compared with antibiotics alone.

CONCLUSION. This study establishes proof of concept for exebacase and direct lytic agents as potential therapeutics and supports conduct of a confirmatory study focused on exebacase to treat MRSA BSIs.

TRIAL REGISTRATION. Clinicaltrials.gov NCT03163446.

FUNDING. ContraFect Corporation.

Conflict of interest: VGF reports grants to his institution and personal consultancy fees from Contrafect. In addition, VGF reports grant/research support from MedImmune, Cerexa/Forest/Actavis/Allergan, Pfizer, Advanced Liquid Logics, Theravance, Novartis, Cubist/Merck, Medical Biosurfaces, Locus, Affinergy, Contrafect, Karius, Genentech, Regeneron, and Basilea, as well as paid consultancies from Pfizer, Novartis, Galderma, Novadigm, Durata, Debiopharm, Genentech, Achaogen, Affinium, Medicines Co., Cerexa, Tetraphase, Trius, MedImmune, Bayer, Theravance, Cubist, Basilea, Affinergy, Janssen, xBiotech, Contrafect, Regeneron, Basilea, and Destiny, and royalties from UpToDate. VGF was a cochair with Merck for the V710 Vaccine; has received educational fees from Green Cross, Cubist, Cerexa, Durata, Theravance, and Debiopharm; and has a patent pending for sepsis diagnostics. AFD reports personal consulting fees from ContraFect, Achaogen, IterumTx, Paratek, Nabriva, Wockhardt, UTILITY, Zavante, Tetraphase, Theravance, and Cempra. JLD reports personal consulting fees from ContraFect. RS is an employee of ContraFect and has US Patent No. 9.889,181 issued and US Patent No. 9,499,594 issued. RP is an employee of ContraFect. LJP reports grants from ContraFect. AB reports grants from ContraFect and personal consulting fees from Theravance, Biopharma, and Allergan. DE reports grants from ContraFect and Merck, as well as grants and personal consulting fees from Tetraphase and AtoxBio. GJM reports grants from Contrafect, as well as grants and personal consulting fees from Nabriva. MER reports grants from ContraFect and Magnolia, and personal consulting fees from Citius and $3 \mathrm{M}$. RW reports personal consulting fees from Contrafect, Pulmonx, Roche, Spiration, Sunovion, Merck, Circassia, Pneuma, Verona, Mylan/Theravance, Propeller Health, AbbVie, Novartis, and Kiniksa; grants from Pearl Therapeutics and Sanofi-Aventis; and grants and personal consulting fees from AstraZeneca/Medimmune/Pearl, Boehringer Ingelheim, and CSK. GRC reports personal consulting fees from Contrafect, Arsanis, Medtronic, Melinta, Motif, Paratek, Regeneron, SCPharma, Shionogi, Tetraphase, and the Medicines Company. MZ reports personal consulting fees from ContraFect and grants from Pfizer, Merck, Medimmune, and Genetech. PSD reports grants from ContraFect. CC is an employee of ContraFect.

Copyright: () 2020, American Society for Clinical Investigation.

Submitted: January 21, 2020; Accepted: March 31, 2020; Published: June 8, 2020.from Nabriva.

Reference information: J Clin Invest. 2020;130(7):3750-3760. https://doi.org/10.1172/JCI136577. 


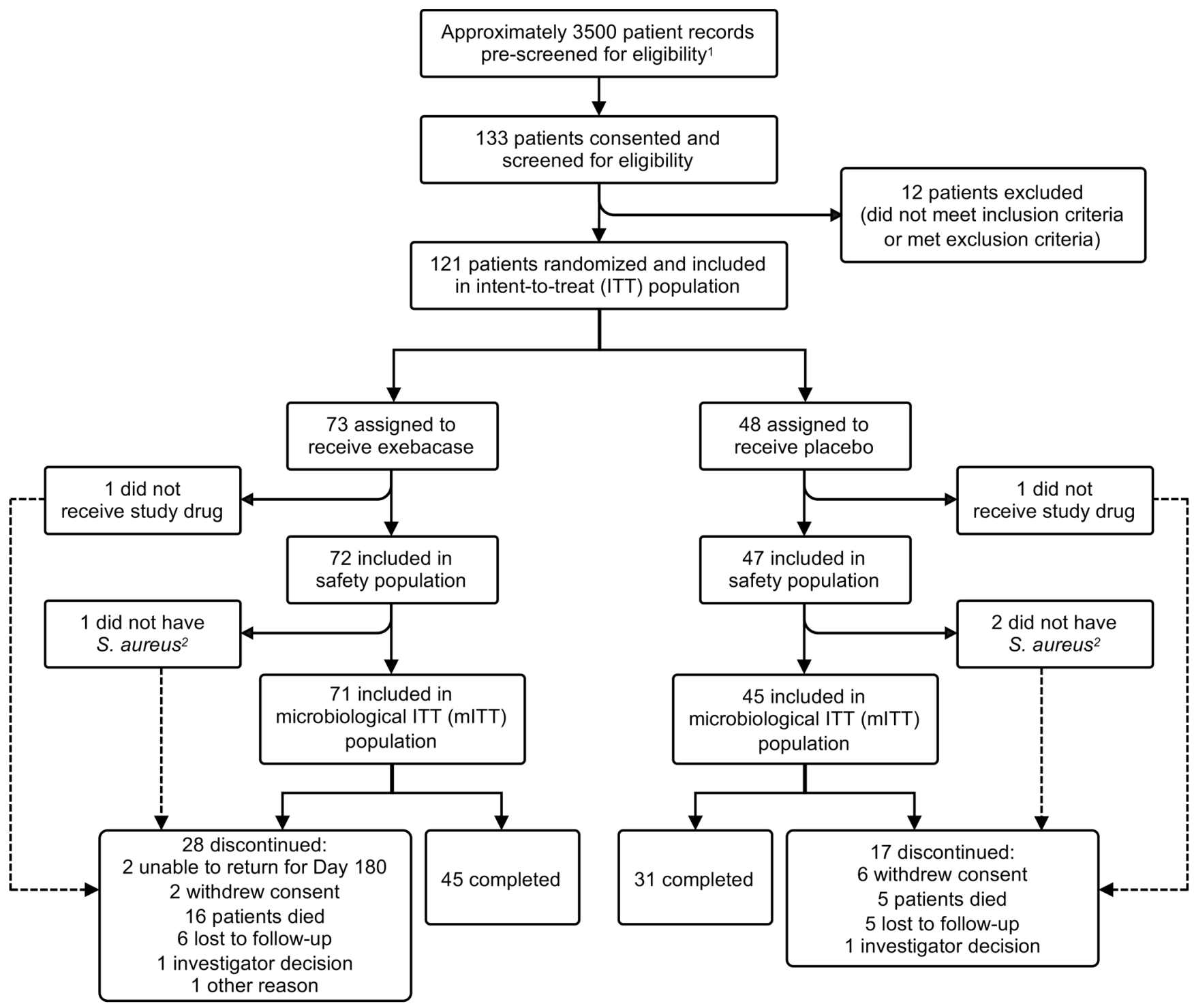

Figure 1. Patient disposition. 'The reasons prescreened patients were deemed to be ineligible are summarized in Supplemental Table 4. ${ }^{2}$ The local laboratory identified Gram-positive cocci in clusters on Gram stain plus positive direct-tube coagulase test and the patients were enrolled; however, the central laboratory subsequently determined that the isolates were Staphylococcus epidermidis.

\section{Introduction}

Complicated Staphylococcus aureus bloodstream infections (BSIs) cause substantial morbidity and mortality (1), which is highest for methicillin-resistant $S$. aureus (MRSA) BSIs (2-5). Mortality rates for patients with $S$. aureus BSI have not changed significantly for decades despite new antibiotics with activity against MRSA (6-8). Hence, there is an urgent need for novel approaches to improve clinical outcomes for $S$. aureus BSI, particularly MRSA.

Exebacase, an antistaphylococcal lysin, is an entirely new antibacterial treatment modality $(9,10)$. As a peptidoglycan hydrolase, recombinantly produced as a purified protein, exebacase results in rapid, pathogen-targeted bacteriolysis, potent biofilm eradication, synergy with antibiotics, low propensity for resistance, and the potential to suppress antibiotic resistance when used together with antibiotics (9-12). Exebacase represents a first-in-field, firstin-class, nonantibiotic antimicrobial direct lytic agent with the potential to improve clinical outcomes of $S$. aureus BSI. Here, we report the safety and efficacy of exebacase used in addition to standard antibiotic therapy to treat $S$. aureus BSI including endocarditis in a superiority-design, proof-of-concept study.

\section{Results}

Trial population. This randomized, double-blind, placebo-controlled, superiority-design, first-in-patient, proof-of-concept study (clinicaltrials.gov identifier: NCT03163446) was conducted at 42 sites in 11 countries between May 2017 and March 2019. A total of 3729 patients were prescreened for eligibility (reasons patients were deemed ineligible are summarized in Supplemental Table 4), of which 121 patients were randomized (intent-to-treat [ITT] population), 119 patients received study drug (exebacase or placebo) (safety population), and 116 patients had confirmed $S$. aureus BSI and were included in the primary efficacy analysis population 
Table 1. Baseline disease characteristics and risk factors/comorbidities (mITT population)

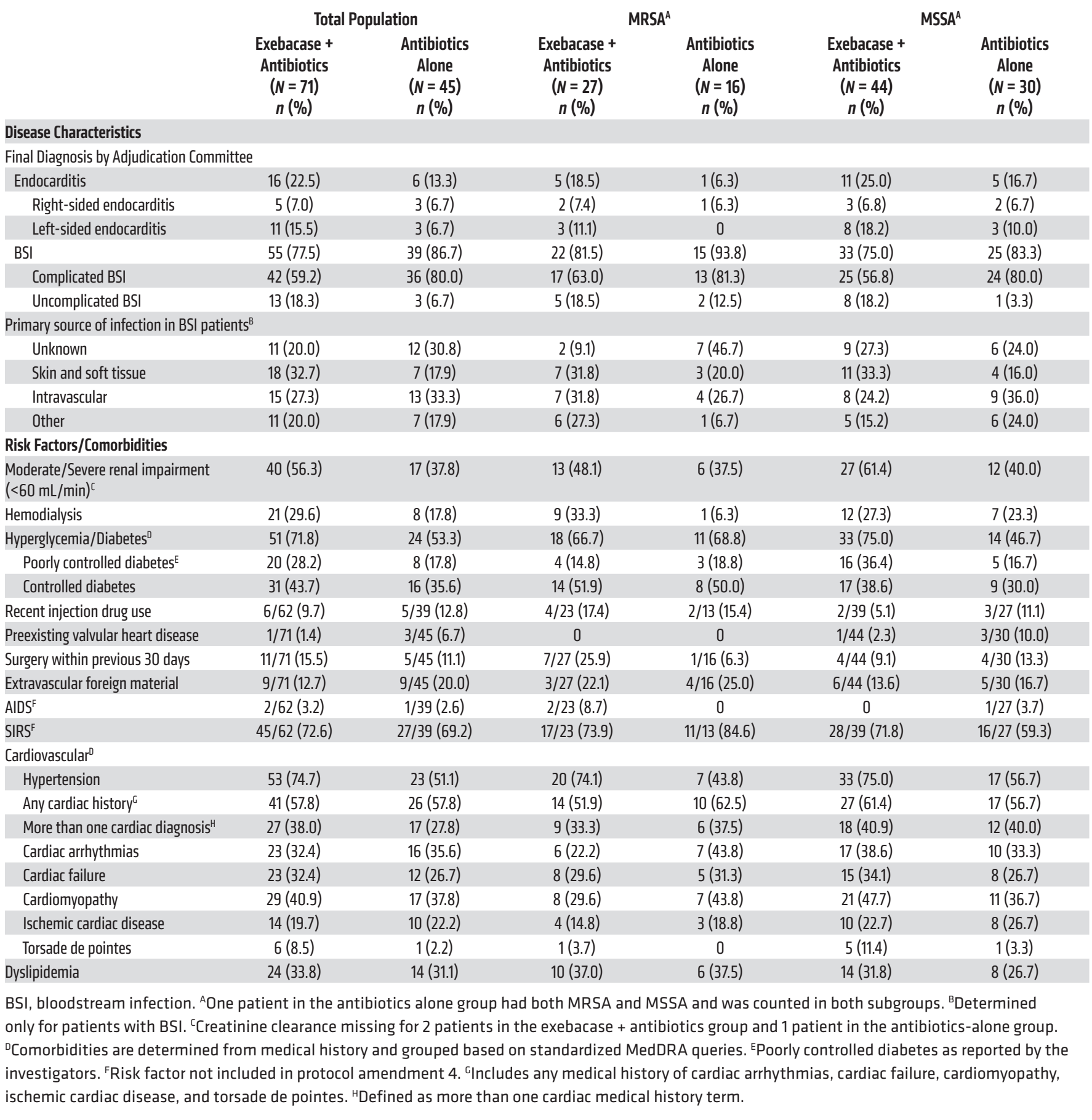

(the microbiological intent-to-treat [mITT] population) (Figure 1). The majority of patients were enrolled in the United States (79.3\%). Small numbers of patients were enrolled in each of the other 10 countries. The average patient was approximately 56 years of age, white (68.1\%), and male (67.2\%).

Approximately one-third of patients in both treatment groups had MRSA (Table 1), resulting in 27 and 16 patients in the MRSA subgroup in the exebacase + antibiotics group and the antibioticsalone group, respectively. More than twice as many patients in the exebacase + antibiotics group compared with the antibioticsalone group had left-sided endocarditis (15.5\% vs. $6.7 \%$ ) and uncomplicated BSI (18.3\% vs. 6.7\%). Renal and cardiovascular comorbidities were also more common in the exebacase + antibiotics group compared with the antibiotics-alone group: $56.3 \%$ and $37.8 \%$ had moderate to severe renal insufficiency, $71.8 \%$ and $53.3 \%$ had diabetes, and $38.0 \%$ and $27.8 \%$ had more than one baseline cardiac diagnosis, respectively. Among patients with BSI, the most common source of infection was skin and 
Table 2. Standard-of-care antibiotics received (mITT population)

\begin{tabular}{|c|c|c|c|c|c|c|}
\hline & \multicolumn{2}{|c|}{ Total Population } & \multicolumn{2}{|c|}{$\mathrm{MRSA}^{\mathrm{A}}$} & \multicolumn{2}{|c|}{ MSSA $^{A}$} \\
\hline & $\begin{array}{c}\text { Exebacase + } \\
\text { Antibiotics } \\
(N=71) \\
n(\%)\end{array}$ & $\begin{array}{c}\text { Antibiotics } \\
\text { Alone } \\
(N=45) \\
n(\%)\end{array}$ & $\begin{array}{c}\text { Exebacase + } \\
\text { Antibiotics } \\
(N=27) \\
n(\%)\end{array}$ & $\begin{array}{c}\text { Antibiotics } \\
\text { Alone } \\
(N=16) \\
n(\%)\end{array}$ & $\begin{array}{c}\text { Exebacase + } \\
\text { Antibiotics } \\
(N=44) \\
n(\%)\end{array}$ & $\begin{array}{c}\text { Antibiotics } \\
\text { Alone } \\
(N=30) \\
n(\%)\end{array}$ \\
\hline \multicolumn{7}{|c|}{ Standard-of-Care Antibiotic Exposure Through Day $14^{\mathrm{B}}$} \\
\hline Daptomycin & $5(11.1)$ & $5(7.0)$ & $5(18.5)$ & $3(18.8)$ & 0 & $3(10.0)$ \\
\hline Vancomycin & $24(33.8)$ & $17(37.8)$ & $21(77.8)$ & $13(81.3)$ & $3(6.8)$ & $4(13.3)$ \\
\hline Mean (SD) & $36.0(24.88)$ & $32.7(17.07)$ & $40.2(33.38)$ & $33.4(17.84)$ & $33.5(18.16)$ & $32.9(17.38)$ \\
\hline Median & 31.0 & 30.0 & 31.0 & 35.0 & 30.5 & 30.0 \\
\hline Minimum, maximum & 5,184 & 4,94 & 7,184 & 2,59 & 5,91 & 4,94 \\
\hline \multicolumn{7}{|c|}{ Duration of Standard-of-Care Antibiotics from Start of Study Drug (Days) $)^{D}$} \\
\hline Mean (SD) & $33.3(24.92)$ & $30.5(17.01)$ & $36.6(33.62)$ & $31.3(17.98)$ & $31.5(18.20)$ & $30.8(17.16)$ \\
\hline \multicolumn{7}{|c|}{$\begin{array}{l}\text { regardless of any changes in antibiotic agent and/or interruptions. Days of antibiotic from start of study drug to last antibiotic } \\
\text { iy changes in antibiotic agent and/or interruptions. }\end{array}$} \\
\hline
\end{tabular}

soft tissue in the exebacase + antibiotics group and intravascular (hemodialysis access or other catheter) in the antibiotics-alone group. In the methicillin-sensitive $S$. aureus (MSSA) subgroup, $8(18.2 \%)$ patients in the exebacase + antibiotics group compared with $3(10.0 \%)$ patients in the antibiotics-alone group had left-sided endocarditis. There were other clinically important differences between the exebacase + antibiotics and antibioticsalone groups with respect to baseline comorbidities, including moderate to severe renal insufficiency (61.4\% vs. $40.0 \%)$, poorly controlled diabetes (36.4\% vs. $16.7 \%)$, and hypertension $(75.0 \%$ vs. $56.7 \%)$.

All patients in the mITT population received an antibiotic to which the baseline pathogen was susceptible within 2 days of study drug administration. Vancomycin and $\beta$-lactams were the most frequently used antibiotics through day 14 . The median duration of antibiotic therapy from the start of study drug was the same in both treatment groups (29 days, range: 2 to 181 days and 2 to 91 days in the exebacase + antibiotics and antibiotics-alone groups, respectively) (Table 2).

Efficacy analyses. In the mITT population, $70.4 \%$ of the exebacase + antibiotics and $60.0 \%$ of the antibiotics-alone groups were clinical responders on day 14 (difference $=10.4,90 \%$ CI $[-6.3,27.2]$, $P=0.31$ ) (Table 3). In the prespecified exploratory MRSA subgroup, the clinical responder rate on day 14 was 42.8 percentage points higher among exebacase-treated patients compared with those who received antibiotics alone $(74.1 \%$ vs. $31.3 \%$, difference $=42.8$, $90 \%$ CI $[14.3,71.4]$, ad hoc $P=0.01)$. Responder rates in the MSSA subgroup were similar between treatment groups. There was 1 patient in the antibiotics-alone group who had both MRSA and MSSA and was counted in each subgroup for the analyses. A sensitivity analysis was completed whereby this patient was excluded from the MRSA and MSSA subgroup analyses. Because this patient was a nonresponder, the responder rate in the antibiotics- alone group increased slightly; however, the conclusions were unchanged. The exploratory analysis of clinical outcome on day 14 in the clinically evaluable population is provided in Supplemental Table 1 (supplemental material available online with this article; https://doi.org/10.1172/JCI136577DS1).

The disproportionately high number of left-sided endocarditis patients randomized to exebacase appeared to affect the efficacy analysis due to the inherent lethality of this disease. An ad hoc analysis of patients with BSI/right-sided endocarditis (i.e., excluding left-sided endocarditis) (Figure 2) found $80.0 \%$ and $59.5 \%$ of patients in the exebacase + antibiotics and antibiotics-alone groups, respectively, were clinical responders (difference $=20.5,90 \% \mathrm{CI}[3.4,37.6]$, ad hoc $P=0.03$ ). Results in MRSA patients with BSI/right-sided endocarditis were similar to the overall MRSA population.

The clinical response pattern observed on day 14 persisted at subsequent time points in both the mITT population and MRSA and MSSA subgroups (Table 3). A bar graph showing clinical outcome on days 7 and 14, which occurred 28 days after the end of treatment with antibiotics (EOT), and test of cure (TOC) is provided in Supplemental Figure 1.

Microbiological response was similar between the exebacase + antibiotics and antibiotics-alone groups on days 7 (83.1\% vs. $86.7 \%$ ) and 14 (90.1\% vs. $84.4 \%)$. In the MRSA subgroup, microbiological response on day 14 in the exebacase + antibiotics and antibiotics-alone groups was $92.6 \%$ versus $75.0 \%$, and in patients with MSSA, $88.6 \%$ versus $90.0 \%$. All patients that were clinical responders on day 14 had negative blood cultures by day 14 . The exploratory analysis of microbiological response on days 7 and 14 in the microbiologically evaluable population is provided in Supplemental Table 2.

Kaplan-Meier curves of time to symptom resolution, time to defervescence, and time to clearance of bacteremia are in Supplemental Figures 2, 3, and 4, respectively. 
Table 3. Clinical outcome throughout the study assessed by adjudication committee by MRSA and MSSA subgroup (mITT population)

\begin{tabular}{|c|c|c|c|c|c|c|}
\hline & \multicolumn{2}{|c|}{ Total Population } & \multicolumn{2}{|c|}{$\mathrm{MRSA}^{\mathrm{A}}$} & \multicolumn{2}{|c|}{ MSSA $^{A}$} \\
\hline & $\begin{array}{c}\text { Exebacase + } \\
\text { Antibiotics } \\
(N=71) \\
n(\%)\end{array}$ & $\begin{array}{c}\text { Antibiotics } \\
\text { Alone } \\
(N=45) \\
n(\%)\end{array}$ & $\begin{array}{c}\text { Exebacase + } \\
\text { Antibiotics } \\
(N=27) \\
n(\%)\end{array}$ & $\begin{array}{c}\text { Antibiotics } \\
\text { Alone } \\
(N=16) \\
n(\%)\end{array}$ & $\begin{array}{c}\text { Exebacase + } \\
\text { Antibiotics } \\
(N=44) \\
n(\%)\end{array}$ & $\begin{array}{c}\text { Antibiotics } \\
\text { Alone } \\
(N=30) \\
n(\%)\end{array}$ \\
\hline \multicolumn{7}{|c|}{ Day 14 (Primary Outcome) } \\
\hline Responder $^{\mathrm{B}}$ & $50(70.4)$ & $27(60.0)$ & $20(74.1)$ & $5(31.3)$ & $30(68.2)$ & $22(73.3)$ \\
\hline Difference $(90 \% \mathrm{Cl})^{\mathrm{C}}$ & \multicolumn{2}{|c|}{$10.4[-6.3,27.2]$} & \multicolumn{2}{|c|}{$42.8[14.3,71.4]$} & \multicolumn{2}{|c|}{$-5.2[-25.6,15.3]$} \\
\hline Nonresponder & $18(25.4)$ & $13(28.9)$ & $4(14.8)$ & $8(50.0)$ & $14(31.8)$ & $6(20.0)$ \\
\hline Indeterminate & $3(4.2)$ & $5(11.1)$ & $3(11.1)$ & $3(18.8)$ & 0 & $2(6.7)$ \\
\hline \multicolumn{7}{|l|}{ Secondary Outcomes } \\
\hline \multicolumn{7}{|l|}{ Day 7} \\
\hline Responder $^{B}$ & $51(71.8)$ & $31(68.9)$ & $18(66.7)$ & $7(43.8)$ & $33(75.0)$ & $25(83.3)$ \\
\hline Nonresponder & $17(23.9)$ & $11(24.4)$ & $7(25.9)$ & $8(50.0)$ & $10(22.7)$ & $2(10.0)$ \\
\hline Nonresponder & $22(31.0)$ & $13(28.9)$ & $8(29.6)$ & $8(50.0)$ & $14(31.8)$ & $6(20.0)$ \\
\hline Indeterminate & $5(7.0)$ & $4(8.9)$ & $5(18.5)$ & $1(6.3)$ & 0 & $3(10.0)$ \\
\hline \multicolumn{7}{|l|}{ TOC } \\
\hline Responder & $39(54.9)$ & $24(53.3)$ & $13(48.1)$ & $5(31.3)$ & $26(59.1)$ & $19(63.3)$ \\
\hline Nonresponder & $25(35.2)$ & $15(33.3)$ & $8(29.6)$ & $9(56.3)$ & $17(38.6)$ & $7(23.3)$ \\
\hline Indeterminate & $7(9.9)$ & $6(13.3)$ & $6(22.2)$ & $2(12.5)$ & $1(2.3)$ & $4(13.3)$ \\
\hline
\end{tabular}

$\mathrm{CI}$, confidence interval; EOT, end of treatment with antibiotics; TOC, test of cure. ${ }^{\mathrm{A}}$ One patient in the antibiotics alone group had both MRSA and MSSA

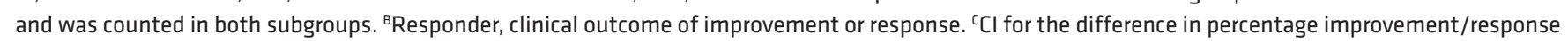
between exebacase + antibiotics and antibiotics-alone groups calculated using a continuity corrected $Z$ statistic. ${ }^{D} P$ value is based on Fisher's exact test.

Safety analyses. The incidence of treatment-emergent adverse events (TEAEs) and serious TEAEs was similar in both groups (Table 4). No TEAEs of hypersensitivity to exebacase were reported, and no TEAEs resulted in withdrawal of study drug. One serious TEAE, which occurred on day 30 after exebacase dosing, was considered related to study drug by an investigator. The 30-day all-cause mortality rate was $9.7 \%$ (7 of 72 ) and $12.8 \%$ (6 of 47 ) in the exebacase + antibiotics and antibiotics-alone groups, respectively. In the MRSA subgroup, the 30-day all-cause mortality rate was $3.7 \%$ ( 1 of 27 ) in the exebacase + antibiotics group and $25.0 \%$ (4 of 16 ) in the antibiotics-alone group (difference $=-21.3,90 \%$ CI $[-45.1,2.5]$, ad hoc $P=0.06$ ). Through TOC, which occurred 28 days after the EOT, mortality rates were $19.4 \%$ (14 of 72 ) and $14.9 \%$ (7 of 47 ) in the exebacase + antibiotics and antibiotics-alone groups, respectively (difference $=4.6,90 \%$ CI $[-8.7,17.8]$, ad hoc $P=0.63)$; a similar trend was observed through day 180 .

Immunogenicity. At baseline, $20.8 \%$ and $14.9 \%$ of patients had preexisting exebacase cross-reactive anti-drug antibodies (ADAs) in the exebacase + antibiotics and antibiotics-alone groups, respectively (Table 5). Clinical responder rates on day 14 were similar among exebacase patients who were $\mathrm{ADA}^{+}$and $\mathrm{ADA}^{-}$ at baseline (73.3\% and $69.6 \%$, respectively), as were TEAE rates (100.0\% and $91.2 \%$, respectively). Of patients who were $\mathrm{ADA}^{-}$at baseline and had evaluable post-dose ADA samples, $71.2 \%$ (37 of 52 ) of exebacase-treated patients and $25.0 \%$ (8 of 32) in the antibiotics-alone group developed treatment-emergent ADAs.
One patient had detectable, low-titer exebacase cross-reactive $\operatorname{IgE}$ at baseline, which remained low after dosing with exebacase. Six patients who received exebacase and none who received antibiotics alone developed treatment-emergent IgE, which were of low titer and transient.

Health resource utilization. Among US patients with MRSA who were alive at hospital discharge, the median length of stay was 6 days in the exebacase + antibiotics group compared with 10 days in the antibiotics-alone group (Table 6). All-cause 30-day readmissions occurred in $16.0 \%$ and $30.8 \%$ of patients in the exebacase + antibiotics and antibiotics-alone groups, respectively. Among US patients with MSSA who were alive at hospital discharge, the median length of stay was 8 and 7 days in the exebacase + antibiotics and antibiotics-alone groups, respectively; all-cause 30 -day readmissions occurred in $27.6 \%$ and $43.5 \%$ of patients in the exebacase + antibiotics and antibiotics-alone groups, respectively.

\section{Discussion}

Exebacase, a first-in-class direct lytic agent, is an entirely new modality for treatment of serious infections caused by $S$. aureus and a member of a new class of nonantibiotic antimicrobials known as lysins (cell wall hydrolase enzymes), which may represent the postantibiotic generation of treatments $(9,10)$.

This first-in-patient, proof-of-concept study tested the utility of exebacase as adjunctive therapy to improve clinical outcomes for $S$. aureus BSI including endocarditis. Because exebacase was 


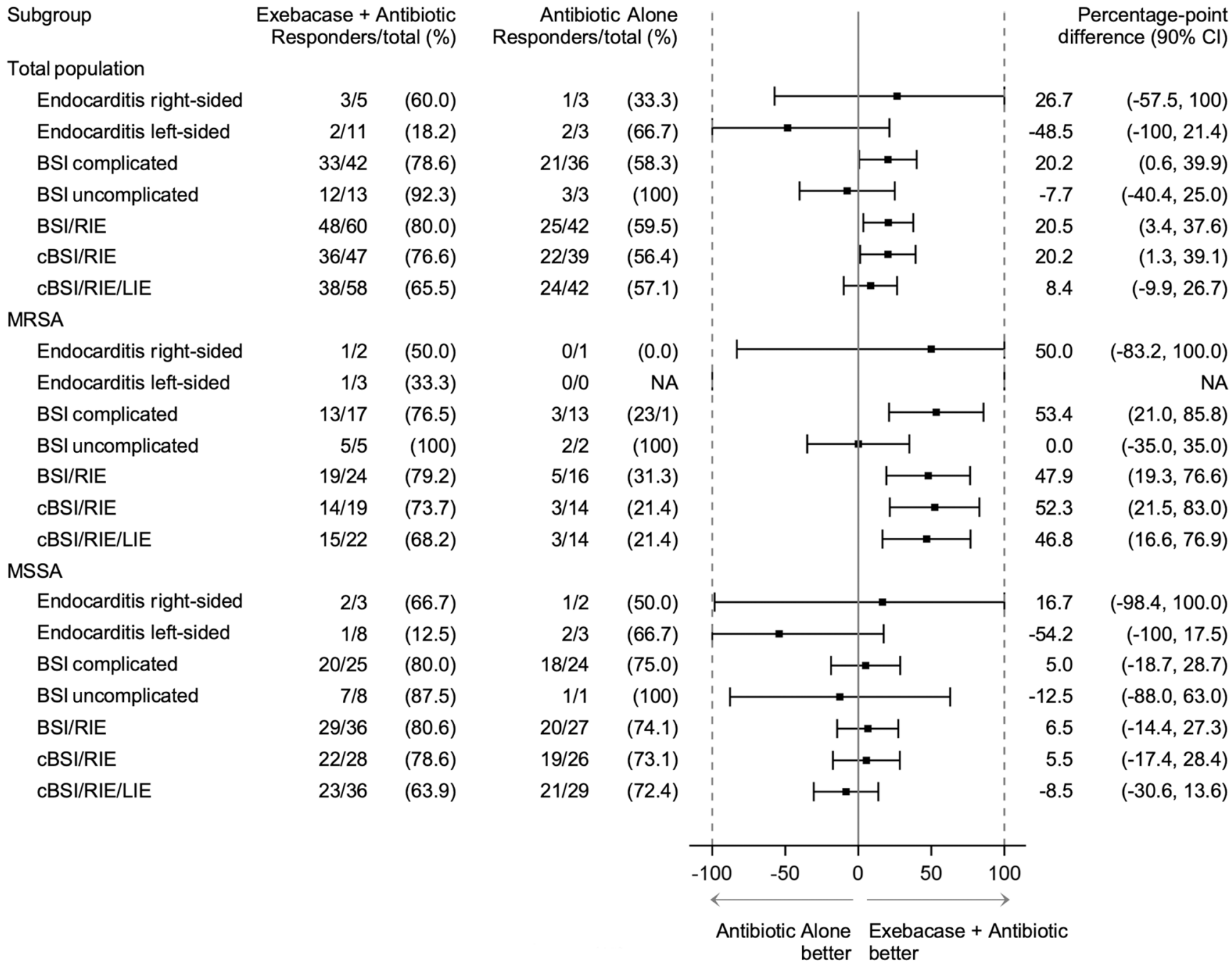

Figure 2. Forest plot of clinical responders by diagnosis. BSI, bloodstream infection; cBSI, complicated bloodstream infection; Cl, confidence interval; LIE, left-sided endocarditis; RIE, right-sided endocarditis; NA, not available as there was no observation in the antibiotic-alone group.

added to standard-of-care antibiotics, this study used a superiority design uncommon in contemporary antibiotic drug development, which typically compares investigational antibiotic versus standard antibiotic in a noninferiority design. While a treatment difference of 10 percentage points was observed in the mITT population on day 14 (70.4\% vs. $60.0 \%)$, treatment with exebacase was associated with a 42.8 percentage point higher clinical responder rate in the MRSA subgroup at the primary day 14 efficacy time point $(74.1 \%$ vs. $31.3 \%)$. The higher responder rates among MRSA patients that received exebacase were sustained at later time points and are supported by reductions in length-of-stay and readmission rates. Responder rates in the MSSA subgroup were similar between treatment groups. The low responder rate of $31.3 \%$ among MRSA patients in the placebo group is consistent with historically worse outcomes with MRSA, compared with MSSA, and allows for the larger treatment difference in patients with MRSA. Based on in vitro microbiological studies and contemporary surveillance studies $(13,14)$, which demonstrate similar activity of exebacase against MRSA and MSSA, there is no evidence of inherent underlying biological differences in the activity of exebacase against MRSA and MSSA. However, while exebacase exhibited no biological differences by itself against MRSA and MSSA, the biological effects of antibiotics used to treat MRSA and MSSA (e.g., vancomycin vs. $\beta$-lactam) to which exebacase was added are very different. Therefore, it is possible that the additive effect of exebacase in patients with MRSA may be due to the drugs to which it is added. The differences in responder rates in the MSSA subgroup may have also been influenced by differences in underlying serious comorbidities and the distribution of left-sided endocarditis between treatment groups.

The results of this study have several key implications. Complicated S. aureus BSIs are serious, common, and potentially lethal infections (15), and MRSA has been identified as a serious threat by both the Centers for Disease Control and the World Health Organization $(16,17)$. The introduction of vancomycin, a major advance in the treatment of MRSA bacteremia, was over 60 years ago. Daptomycin, the newest drug developed for S. aureus BS I, is over 13 years old and was approved based on noninferiority to older antibiotics, with MRSA clinical success rates of $44.4 \%$ for daptomycin and $31.8 \%$ for vancomycin (18). Subsequent attempts 
Table 4. Overview of adverse events (safety population)

\begin{tabular}{|c|c|c|}
\hline & $\begin{array}{c}\text { Exebacase + } \\
\text { Antibiotics } \\
(N=72) \\
n(\%)\end{array}$ & $\begin{array}{c}\text { Antibiotics } \\
\text { Alone } \\
(N=47) \\
n(\%)\end{array}$ \\
\hline \multicolumn{3}{|l|}{ Number of patients with: } \\
\hline TEAE through day 7 & $48(66.7)$ & $30(63.8)$ \\
\hline TEAE through TOC & $64(88.9)$ & $40(85.1)$ \\
\hline \multicolumn{3}{|c|}{ Events occurring in $\geq 8 \%$ through TOC in either treatment group } \\
\hline Urinary tract infection & $8(11.1)$ & $6(12.8)$ \\
\hline Constipation & $9(12.5)$ & $5(10.6)$ \\
\hline Diarrhea & $8(11.1)$ & $3(6.4)$ \\
\hline Headache & $7(9.7)$ & $4(8.5)$ \\
\hline Anemia & $6(8.3)$ & $4(8.5)$ \\
\hline Cardiac murmur & $6(8.3)$ & $1(2.1)$ \\
\hline Edema peripheral & $6(8.3)$ & $4(8.5)$ \\
\hline Nausea & $6(8.3)$ & $3(6.4)$ \\
\hline Death NOS & $1(1.4)$ & $4(8.5)$ \\
\hline Abdominal pain & 0 & $5(10.6)$ \\
\hline Serious TEAE through TOC & $34(47.2)$ & $23(48.9)$ \\
\hline Serious TEAE through Day 180 & $45(62.5)$ & $28(59.6)$ \\
\hline TEAE related to study drug & $8(11.1)$ & $4(8.5)$ \\
\hline Serious TEAE related to study drug & $1(1.4)$ & 0 \\
\hline $\begin{array}{l}\text { TEAE leading to study drug } \\
\text { discontinuation/withdrawal }\end{array}$ & 0 & 0 \\
\hline $\begin{array}{l}\text { TEAE of hypersensitivity to } \\
\text { exebacase }\end{array}$ & 0 & NA \\
\hline Death through day 30 & $7(9.7)$ & $6(12.8)$ \\
\hline Death through TOC ${ }^{A}$ & $14(19.4)$ & $7(14.9)$ \\
\hline Death through day 180 & $17(23.6)$ & $9(19.1)$ \\
\hline
\end{tabular}

TEAE, treatment-emergent adverse event; TOC, test of cure; NA, not applicable; NOS, not otherwise specified. Denominator is all patients in the safety population within each treatment group. ${ }^{A}$ All deaths through TOC occurred before day 60 , so this represents day 60 mortality.

to develop new antibiotics for $S$. aureus bacteremia have failed (19, 20). The addition of adjunctive agents such as immunotherapeutics (21-24) or antibiotics (e.g., gentamicin, rifampin, or $\beta$-lactams; refs. 25-27) to standard therapy for $S$. aureus or MRSA BSI has generally shown disappointing results in clinical trials, with the exception of a recent open-label pilot study of the initial combination of daptomycin and ceftaroline, which showed potentially promising results to be confirmed in a larger randomized clinical trial (28). Thus, the urgent need for effective new treatments for $S$. aureus BSI, and MRSA BSI in particular, remains unaddressed.

Based on the novel properties of lysins, which are complementary to and synergistic with antibiotics $(9-12,33)$, and the unmet need for agents to improve clinical outcomes for $S$. aureus BSI/endocarditis associated with conventional antibiotics alone, exebacase is being developed as adjunctive therapy. The current study is the first to our knowledge to show promising improvements in clinical outcomes among patients with $S$. aureus BSI who received adjunctive lysin therapy. This improvement was particularly marked in the prespecified exploratory MRSA subgroup. Exebacase was generally safe and well tolerated, with AEs consistent with those expected in critically ill, hospitalized patients with potentially life-threatening
S. aureus BSI, including endocarditis and/or underlying comorbid conditions. Overall, the 30-day all-cause mortality rate was $9.7 \%$ in the exebacase-treated group and $12.8 \%$ in the antibiotics-alone group, with a greater difference in the MRSA subgroup $(3.7 \%$ vs. $25.0 \%$ ). These findings are important, considering that 28 -day mortality has been used as a standard for assessment of survival in hospitalized patients with serious infections (e.g., hospitalacquired and ventilator-associated bacterial pneumonia [HABP/ VABP] $)(29,30)$, and is an FDA-recommended endpoint in HABP/ VABP trials (31). All-cause mortality rates in both groups were higher at the TOC time point, which varied widely between patients (up to 180 days after dosing) allowing time for mortality due to medical events unrelated to the infection under study. The TOC all-cause mortality rates of $19.4 \%$ and $14.9 \%$ in the exebacase + antibiotics and antibiotics-alone groups may have been affected by the higher number of comorbidities and patients with left-sided endocarditis in the exebacase-treated group.

Importantly, no hypersensitivity reactions to exebacase were reported, despite the fact that $20 \%$ of exebacase-treated patients had baseline exebacase ADAs. The preexisting ADAs did not affect efficacy or safety outcomes and exebacase does not appear to be sensitizing for allergic hypersensitivity. The presence of baseline antibodies against exebacase may be explained by recent findings that exposures to $S$. aureus (and likely other pathogens, including streptococci) results in human antibody responses against a range of cell wall proteins, including autolysins (32), which would be expected to share common structural motifs and antigenic domains with exebacase. Prior exposures to staphylococci or streptococci (and the generation of antibodies) may occur during the course of infection, or during carriage of these organisms in microbiome environments.

Among US patients with MRSA, exebacase was associated with lower median length of stay and 30-day hospital readmission rates compared with antibiotics alone. This orthogonal analysis further supports the clinical efficacy observed in the MRSA subgroup. While the precise drivers of these reductions in health resource utilization are not known, the hallmark antibacterial actions of exebacase, including rapid bactericidality, eradication of S. aureus biofilms, and potent synergy with antibiotics that have been well described in vivo and in vitro $(9-12,33)$ may have played a role.

A limitation of the study was the relatively small sample size, especially in the MRSA subset, given this was a first-in-patient, proof-of-concept rather than a confirmatory study. The sample size for the MRSA subset was not prespecified since the analysis in this population was an exploratory objective of the protocol. Another limitation was the difference in the proportion of patients with left-sided endocarditis and uncomplicated BSI between treatment groups. The baseline difference in left-sided endocarditis may have affected the efficacy and safety analyses, given that these patients have poor outcomes and generally require surgical intervention. The results in the MSSA subset may also be difficult to interpret given differences between treatment groups in clinically important serious underlying comorbidities. In addition, EOT and TOC were not fixed time points, which may affect the interpretation of the efficacy findings at these time points. The 30-day mortality rates in this study were lower than those seen in cohort studies $(1,6)$, but are similar to mortality 
Table 5. Immunogenicity: treatment-emergent ADA and IgE (safety population)

\begin{tabular}{|c|c|c|}
\hline & $\begin{array}{l}\text { Exebacase }+ \text { Antibiotics } \\
\qquad(N=72)\end{array}$ & $\begin{array}{l}\text { Antibiotics Alone } \\
\qquad(N=47)\end{array}$ \\
\hline Parameter & $n(\%)$ & $n(\%)$ \\
\hline Exebacase $\mathrm{ADA}^{+}$at baseline & $15 / 72(20.8)$ & $7 / 47(14.9)$ \\
\hline Exebacase $\mathrm{ADA}^{-}$at baseline & $57 / 72(79.2)$ & $40 / 47(85.1)$ \\
\hline $\begin{array}{l}\text { Exebacase } \mathrm{ADA}^{-} \text {at baseline and with } \\
\text { NO post-dose sample }\end{array}$ & 5 & 8 \\
\hline $\begin{array}{l}\text { Exebacase } \mathrm{ADA}^{-} \text {at baseline and with at } \\
\text { least } 1 \text { post-dose sample }\end{array}$ & 52 & 32 \\
\hline No treatment-emergent ADA & $15(28.8)$ & $24(75.0)$ \\
\hline Treatment-emergent positive ADA & $37(71.2)$ & $8(25.0)$ \\
\hline Persistence ${ }^{A}$ & $15(28.8)$ & $3(9.4)$ \\
\hline Transient & $3(5.8)$ & $4(12.5)$ \\
\hline $\begin{array}{l}\text { Indeterminate (no day } 180 \\
\text { sample) }\end{array}$ & $19(36.5)$ & $1(3.1)$ \\
\hline IgE $^{+}$at baseline ${ }^{B}$ & $1 / 72(1.4)$ & $0 / 46(0)$ \\
\hline IgE- at baseline $^{B}$ & $71 / 72(98.6)$ & $46 / 46(100)$ \\
\hline $\begin{array}{l}\text { IgE- at baseline and with no post-dose } \\
\text { sample }\end{array}$ & 6 & 9 \\
\hline $\begin{array}{l}\text { IgE- at baseline and with at least } 1 \\
\text { post-dose sample }\end{array}$ & 65 & 37 \\
\hline No treatment-emergent lgE & $56(86.1)$ & $36(97.3)$ \\
\hline Treatment-emergent lgE & $9(13.9)$ & $1(2.7)$ \\
\hline Persistence (positive to day 180) & $3(4.6)$ & 0 \\
\hline $\begin{array}{l}\text { Transient (negative result after } \\
\text { positive result) }\end{array}$ & $3(4.6)$ & $1(2.7)$ \\
\hline $\begin{array}{l}\text { Indeterminate (no result after } \\
\text { single positive result) }\end{array}$ & $3(4.6)$ & 0 \\
\hline
\end{tabular}

ADA, anti-drug antibody. Percentages are based on the number of patients with negative baseline and at least 1 post-baseline sample. Treatment-emergent ADA/IgE was defined as negative ADA/IgE at baseline and emergence of positive ADA/IgE after dosing. ${ }^{A}$ One patient had treatment-emergent ADA that emerged on day 65 and persisted through day 187. One patient had treatment-emergent ADA that emerged on day 62 and persisted through day $176 .{ }^{B}$ One patient in the placebo group was missing the baseline IgE sample.

rates in recent clinical trials of $S$. aureus bacteremia $(18,20,27)$. This difference in mortality rates in cohort studies versus interventional trials reflects the intrinsic difference in the purpose of clinical medicine versus clinical trials. Because clinical trials primarily seek to evaluate the efficacy of a product, stringent inclusion/exclusion criteria are in place to exclude those patients who are likely to have poor clinical outcome due to factors unrelated to $S$. aureus BSI (e.g., malignancy). Given the unmet medical need to improve the clinical success rates for $S$. aureus/MRSA BSI with antibiotics alone, this study evaluated exebacase used in addition to standard therapy. The efficacy of exebacase as a monotherapy was not evaluated in this study. The potential use of exebacase as monotherapy could be explored, as appropriate, for discrete clinical problems for which standard antibiotic therapy is not available (e.g., resistant pathogens).

In summary, this study establishes proof of concept for exebacase and the emerging new class of direct lytic agents as potential therapeutics for BSI caused by MRSA. Moreover, these data sup- port the testing of exebacase in a confirmatory study with a focus on MRSA. Given the consistently poor outcomes of MRSA BSI treated with standard antibiotic therapy and the long list of failed attempts to develop new treatments, these data suggest that exebacase may be the first tangible opportunity in decades to improve clinical responder rates and reduce mortality for MRSA BSI.

\section{Methods}

Trial design and oversight. An independent Data Safety Monitoring Board reviewed unblinded safety and pharmacokinetic data at prespecified points. Clinical response was assessed by the investigator. An independent, blinded Adjudication Committee adjudicated eligibility, final diagnosis including endocarditis determination, and clinical response. Echocardiograms were adjudicated by a blinded cardiologist at an echocardiography laboratory according to standard methodology (34). S. aureus identification and susceptibility were confirmed by a central microbiology laboratory.

ContraFect Corporation, as study sponsor, designed and conducted the study in collaboration with the principal investigator. ContraFect prepared the statistical analysis plan, conducted the analyses, and interpreted the data in conjunction with the authors. The protocol is included in the supplemental material.

Patient population and treatment. Eligible patients were at least 18 years of age, met screening criteria, and had Gram-positive cocci in clusters on Gram stain plus positive direct-tube coagulase test or blood culture positive for $S$. aureus within 72 hours before randomization. Echocardiography was performed within 3 days of randomization (35). Removable sources of infection (e.g., intravascular line, abscess, dialysis graft) were removed or debrided within 72 hours after randomization. All patients received antibiotics selected by the investigator according to the protocol consisting of semisynthetic penicillins or first-generation cephalosporins for MSSA and vancomycin or daptomycin for MRSA. Patients were randomly assigned in a 3:2 ratio using a blocked randomization scheme to receive a single 2-hour intravenous infusion of blinded study drug (exebacase or placebo). A 3:2 randomization ratio was used so as to expose a larger proportion of patients to exebacase compared with placebo, but also maximize the sample size in the placebo group. Exebacase was dosed at $0.25 \mathrm{mg} / \mathrm{kg}$ based on target attainment studies in animals and phase I data in humans. While the study was ongoing, review of PK data by the Data Safety Monitoring Board resulted in dose adjustment to $0.12 \mathrm{mg} / \mathrm{kg}$ for patients with creatinine clearance of less than $60 \mathrm{~mL} / \mathrm{min}$ and/or age of more than 50 .

Analysis population, endpoints, and assessments. The ITT population included all randomized patients. The safety population included all patients who received study drug. The primary efficacy analysis population, mITT, included all patients with confirmed $S$. aureus BSI who received study drug.

Patient assessments occurred on days 7 and 14, EOT, and TOC 28 days after EOT, with long-term follow-up of immunogenicity and safety on day 180 after study drug dosing.

The primary objectives were to describe safety and tolerability and estimate clinical outcome on day 14 of exebacase + antibiotics compared with antibiotics alone. Day 14 was selected as the primary efficacy endpoint because it was hypothesized that exebacase's novel, rapid mechanism of action and hallmark properties $(9-12,33)$ would lead to a more rapid resolution of clinical signs and symptoms of infection. The day 14 time point allowed for evaluation of the clinical 
Table 6. Health resource utilization in US patients (mITT population)

\begin{tabular}{|c|c|c|c|c|c|c|}
\hline & \multicolumn{2}{|c|}{ All US Patients } & \multicolumn{2}{|c|}{ MRSA ${ }^{A}$} & \multicolumn{2}{|c|}{ MSSA $^{A}$} \\
\hline & $\begin{array}{c}\text { Exebacase + } \\
\text { Antibiotics } \\
(N=57) \\
n(\%)\end{array}$ & $\begin{array}{c}\text { Antibiotics } \\
\text { Alone } \\
(N=37) \\
n(\%)\end{array}$ & $\begin{array}{c}\text { Exebacase + } \\
\text { Antibiotics } \\
(N=26) \\
n(\%)\end{array}$ & $\begin{array}{c}\text { Antibiotics } \\
\text { Alone } \\
(N=15) \\
n(\%)\end{array}$ & $\begin{array}{c}\text { Exebacase + } \\
\text { Antibiotics } \\
(N=31) \\
n(\%)\end{array}$ & $\begin{array}{c}\text { Antibiotics } \\
\text { Alone } \\
(N=23) \\
n(\%)\end{array}$ \\
\hline In-hospital mortality & $3(5.3)$ & $2(5.4)$ & $1(3.9)$ & $2(13.3)$ & $2(6.5)$ & 0 \\
\hline Patients discharged alive & 54 & 35 & 25 & 13 & 29 & 23 \\
\hline \multicolumn{7}{|c|}{ Number of hospital days from dose of study drug to hospital discharge } \\
\hline Median & 7.0 & 7.0 & 6.0 & 10.0 & 8.0 & 7.0 \\
\hline Minimum, maximum & 2,69 & 2,51 & 2,69 & 5,51 & 3,66 & 2,46 \\
\hline 30-day all-cause readmission ${ }^{B}$ & $12(22.2)$ & $13(37.1)$ & $4(16.0)$ & $4(30.8)$ & $8(27.6)$ & $10(43.5)$ \\
\hline 30-day S. aureus readmission ${ }^{B}$ & $3(5.6)$ & $2(5.7)$ & $2(8.0)$ & $2(15.4)$ & $1(3.4)$ & 0 \\
\hline
\end{tabular}

effect of exebacase in a superiority-design study with less likelihood of confounding by adverse medical occurrences unrelated to the disease under study that may occur at later time points.

Key secondary and exploratory objectives were to estimate clinical outcome on day 7, EOT, and TOC; estimate microbiological outcome on days 7 and 14; describe clinical outcomes in patients with MRSA and by diagnosis; describe post-dose immunological response to exebacase; and explore health resource utilization. Uncomplicated or complicated BSI and right- or left-sided endocarditis were mutually exclusive diagnoses for the analyses. Clinical response was defined as survival with improvement or resolution of attributable signs and symptoms, and without new signs or symptoms, new foci of infection, change in antibiotics due to nonresponse, complications of $S$. aureus, or further surgery or medical intervention to treat $S$. aureus. Microbiological response was defined as 2 consecutive blood cultures collected on different days yielding no $S$. aureus growth.

Safety assessments included AEs, vital signs, electrocardiograms, and clinical laboratory tests. TEAEs were those with an onset or worsening of severity that occurred at or after administration of study drug through TOC.

Statistics. The study was designed to provide proof of concept and an initial assessment of efficacy and was not considered a confirmatory trial. The sample size of approximately 70 and 45 patients in the exebacase + antibiotics and antibiotics-alone groups, respectively, provided at least $80 \%$ power to detect a treatment difference of 25 percentage points in clinical response on day 14, based on expected clinical responder rates of $60 \%$ in the antibiotics-alone group and $85 \%$ in the exebacase + antibiotics group.

Clinical outcome on day 14, as assessed by the Adjudication Committee, was the primary efficacy variable. For the primary analysis, once a patient was assessed as a clinical nonresponder (i.e., failure) due to death, new metastatic foci, complications or surgery due to $S$. aureus, or change in antibiotics due to nonresponse, the patient was a clinical nonresponder for all subsequent visits through TOC. The clinical responder rates on day 14 were compared between treatment groups using Fisher's exact test. Statistical significance was based on a 2 -sided $\alpha$ level of 0.05 . No adjustment was made for multiple comparisons, as confirmatory inferential analyses were not conducted for secondary outcomes or subgroup analyses of clinical response on day 14 (statistical comparisons were an ad hoc analysis). Two-sided $90 \%$ CIs for the difference in outcome rates between treatment groups were calculated using a continuitycorrected $Z$ statistic.

Study approvals. This trial was conducted in accordance with Good Clinical Practice and the Declaration of Helsinki. The institutional review board (IRB) for each site approved the protocol, and written informed consent was obtained for all patients. The central IRB was Western Institutional Review Board (Puyallup, Washington, USA). A list of the IRBs for each site is provided Supplemental Table 3.

\section{Author contributions}

VGF, CC, AFD, JLD, and RS contributed to the design of the clinical study. AFD performed the statistical analysis. VGF, CC, AFD, JLD, RS, and RP contributed to data interpretation. VGF was the principal investigator for the study. LJP, AB, DE, GJM, and MER were investigators and enrolled patients into the study. RW, GRC, and MZ were adjudicators for the study. PSD interpreted echocardiograms. JLD was responsible for writing the manuscript. All authors reviewed and edited the manuscript.

\section{Acknowledgments}

We thank the patients who participated in this study; the members of the Data Safety Monitoring Committee and advisors; the Investigators who enrolled patients in this study (D. Altman, New York, New York, USA; A. Antoniadou, Athens, Greece; J. Baddley, Birmingham, Alabama, USA; I. Baird, Columbus, Ohio, USA; A.M. Buitrago, Idaho Falls, Idaho, USA; G. Daikos, Athens, Greece; S. Dhar, Detroit, Michigan, USA; A. Garnero, Toulon, France; C. Gianatiempo, Englewood, New Jersey, USA; L. Gonzales, Santa Rosita, Guatemala; R. Harper, Sacramento, California, USA; M. Huilcaman, Santiago, Chile; F. Jacobs, Brussels, Belgium; T. Kerkering, Roanoke, Virginia, USA; B. Knoll, Westchester, New York, USA; C. Kraft, Atlanta, Georgia, USA; S. Liang, St. Louis, Missouri, USA; T. Liesching, Burlington, Massachusetts, USA; A. Limaye, Seattle, Washington, USA; E. Maillart, Brussels, Belgium; B. Menzaghi, 
Varese, Italy; L. Morrow, Omaha, Nebraska, USA; K. Mullane, Chicago, Illinois, USA; R. Nanchal, Milwaukee, Wisconsin, USA; W. Nseir, Nazareth, Israel; A. Olivia, Guatemala City, Guatemala; J. Pullman, Butte, Montana, USA; L. Reill, Berlin, Germany; J. Reinhardt, Newark, Delaware, USA; M. Scarborough, Oxford, United Kingdom; J. Slim, Newark, New Jersey, USA; S. Tiberi, London, United Kingdom; J. Vazquez, Augusta, Georgia, USA; M. Virata, New Haven, Connecticut, USA; I. Welters, Merseyside, United Kingdom; J. Wisler, Columbus, Ohio, USA; M. Witzenrath, Berlin, Germany; I. Zabolostskikh, Krasnodar, Russia; the ContraFect Study Team (J. Ambler, D. Anastasiou, J. Boyle, N. Capra, T. Car- abeo, M. Chapnick, J. Chiu, S. Ermlich P. Ghahramani, E. Hershberger); and C. Cheli for editorial assistance with the manuscript.

Address correspondence to: Vance G. Fowler, Jr., Division of Infectious Diseases, Room 315 Hanes Building, 315 Trent Drive, Box 102359, Duke University School of Medicine, Durham, North Carolina 27710, USA. Phone: 919.668.6053; Email: Vance.Fowler@duke.edu.

DE's present address is: OhioHealth Grant Medical Center, Columbus, Ohio, USA.
1. Fowler VG, et al. Clinical identifiers of complicated Staphylococcus aureus bacteremia. Arch Intern Med. 2003;163(17):2066-2072.

2. Melzer M, Eykyn SJ, Gransden WR, Chinn S. Is methicillin-resistant Staphylococcus aureus more virulent than methicillin-susceptible S. aureus? A comparative cohort study of British patients with nosocomial infection and bacteremia. Clin Infect Dis. 2003;37(11):1453-1460.

3. Cosgrove SE, Sakoulas G, Perencevich EN, Schwaber MJ, Karchmer AW, Carmeli Y. Comparison of mortality associated with methicillinresistant and methicillin-susceptible Staphylococcus aureus bacteremia: a meta-analysis. Clin Infect Dis. 2003;36(1):53-59.

4. de Kraker ME, et al. Clinical impact of antimicrobial resistance in European hospitals: excess mortality and length of hospital stay related to methicillin-resistant Staphylococcus aureus bloodstream infections. Antimicrob Agents Chemother. 2011;55(4):1598-1605.

5. WHO. Antimicrobial resistance: global report on surveillance 2014. Geneva, Switzerland: WHO; 2014.

6. Souli M, et al. Changing characteristics of Staphylococcus aureus bacteremia: results from a 21-year, prospective, longitudinal study. Clin Infect Dis. 2019;69(11):1868-1877.

7. Tong SY, Davis JS, Eichenberger E, Holland TL, Fowler VG. Staphylococcus aureus infections: epidemiology, pathophysiology, clinical manifestations, and management. Clin Microbiol Rev. 2015;28(3):603-661.

8. Turner NA, et al. Methicillin-resistant Staphylococcus aureus: an overview of basic and clinical research. Nat Rev Microbiol. 2019;17(4):203-218.

9. Schuch R, Khan BK, Raz A, Rotolo JA, Wittekind M. Bacteriophage lysin CF-301, a potent antistaphylococcal biofilm agent. Antimicrob Agents Chemother. 2017;61(7):e02666-16.

10. Indiani C, et al. The antistaphylococcal lysin, CF-301, activates key host factors in human blood to potentiate methicillin-resistant Staphylococcus aureus bacteriolysis. Antimicrob Agents Chemother. 2019;63(4):e02291-18.

11. Schuch R, et al. Combination therapy with lysin CF-301 and antibiotic is superior to antibiotic alone for treating methicillin-resistant Staphylococcus aureus-induced murine bacteremia. J Infect Dis. 2014;209(9):1469-1478.

12. Watson A, Sauve K, Cassino C, Schuch R. Exebacase demonstrates in vitro synergy with a broad range of antibiotics against both methicillinresistant and methicillin-susceptible Staphy- lococcus aureus. Antimicrob Agents Chemother. 2020;64(2):e01885-19.

13. Watson A, Oh JT, Sauve K, Bradford PA, Cassino C, Schuch R. Antimicrobial activity of exebacase (lysin CF-301) against the most common causes of infective endocarditis. Antimicrob Agents Chemother. 2019;63(10):e01078-19.

14. Traczewski M, Oh J, Cassino C, Schuch R. In vitro activity of exebacase (CF-301) against clinical Staphylococcus aureus surveillance isolates from the United States, Europe, and Latin America, 2015-2017. Diagn Microbiol Infect Dis. 2019;95(4):114879.

15. Kourtis AP, et al. Vital Signs: Epidemiology and recent trends in methicillin-resistant and in methicillin-susceptible Staphylococcus aureus bloodstream infections - United States. MMWR Morb Mortal Wkly Rep. 2019;68(9):214-219.

16. CDC. Antibiotic resistance threats in the United States, 2019. https://www.cdc.gov/drugresistance/Biggest-Threats. Accessed April 27, 2020.

17. WHO. Global priority list of antibiotic-resistant bacteria to guide research, discovery, and development of new antibiotics. Geneva, Switzerland: WHO; 2017.

18. Fowler VG, et al. Daptomycin versus standard therapy for bacteremia and endocarditis caused by Staphylococcus aureus. N Engl J Med 2006;355(7):653-665.

19. A phase 3 telavancin Staphylococcus aureus (S. aureus) bacteremia trial. https://clinicaltrials. gov. NCT02208063. Accessed April 27, 2020.

20. Holland TL, et al. Considerations for clinical trials of Staphylococcus aureus bloodstream infection in adults. Clin Infect Dis. 2019;68(5):865-872.

21. Weems JJ, et al. Phase II, randomized, doubleblind, multicenter study comparing the safety and pharmacokinetics of tefibazumab to placebo for treatment of Staphylococcus aureus bacteremia. Antimicrob Agents Chemother. 2006;50(8):2751-2755.

22. Rupp ME, et al. Phase II, randomized, multicenter, double-blind, placebo-controlled trial of a polyclonal anti-Staphylococcus aureus capsular polysaccharide immune globulin in treatment of Staphylococcus aureus bacteremia. Antimicrob Agents Chemother. 2007;51(12):4249-4254.

23. Weisman LE, et al. A randomized study of a monoclonal antibody (pagibaximab) to prevent staphylococcal sepsis. Pediatrics. 2011;128(2):271-279.

24. Otto M. Novel targeted immunotherapy approaches for staphylococcal infection. Expert Opin Biol Ther. 2010;10(7):1049-1059.

25. Cosgrove SE, et al. Initial low-dose gentamicin for Staphylococcus aureus bacteremia and endocarditis is nephrotoxic. Clin Infect Dis. 2009;48(6):713-721.

26. Thwaites GE, et al. Adjunctive rifampicin for Staphylococcus aureus bacteraemia (ARREST): a multicentre, randomised, double-blind, placebo-controlled trial. Lancet. 2018;391(10121):668-678.

27. Tong SYC, et al. Effect of vancomycin or daptomycin with vs without an antistaphylococca $\beta$-lactam on mortality, bacteremia, relapse, or treatment failure in patients with MRSA bacteremia: a randomized clinical trial. JAMA. 2020;323(6):527-537.

28. Geriak M, et al. Clinical data on daptomycin plus ceftaroline versus standard of care monotherapy in the treatment of methicillin-resistant Staphylococcus aureus bacteremia. Antimicrob Agents Chemother. 2019;63(5):e02483-18.

29. Infectious Diseases Society of America (IDSA), American College of Chest Physicians (ACCP), American Thoracic Society (ATS), Society of Critical Care Medicine (SCCM), Spellberg B, Talbot G. Recommended design features of future clinical trials of antibacterial agents for hospital-acquired bacterial pneumonia and ventilator-associated bacterial pneumonia. Clin Infect Dis. 2010;51 suppl 1:S150-S170.

30. Weiss E, Essaied W, Adrie C, Zahar JR, Timsit JF. Treatment of severe hospital-acquired and ventilator-associated pneumonia: a systematic review of inclusion and judgment criteria used in randomized controlled trials. Crit Care. 2017;21(1):162.

31. U.S. Food and Drug Administration. FDA Guidance Documents. Center for Drug Evaluation and Research. Hospital-acquired bacterial pneumonia and ventilator-associated bacterial pneumonia: developing drugs for treatment. https://www. fda.gov/regulatory-information/search-fda-guidance-documents/hospital-acquired-bacterialpneumonia-and-ventilator-associated-bacterial -pneumonia-developing-drugs. Updated August 24, 2018. Accessed April 27, 2020.

32. Romero Pastrana F, et al. Human antibody responses against non-covalently cell wallbound Staphylococcus aureus proteins. Sci Rep. 2018;8(1):3234

33. Oh JT, Cassino C, Schuch R. Postantibiotic and sub-MIC effects of exebacase (lysin CF-301) 
enhance antimicrobial activity against Staphylococcus aureus. Antimicrob Agents Chemother. 2019;63(6):e02616-18.

34. Douglas PS, et al. Echocardiographic imaging in clinical trials: American Society of Echocardi- ography Standards for echocardiography core laboratories: endorsed by the American College of Cardiology Foundation. J Am Soc Echocardiogr. 2009;22(7):755-765.

35. Baddour LM, et al. Infective endocarditis in adults: diagnosis, antimicrobial therapy, and management of complications: a scientific statement for healthcare professionals from the American Heart Association. Circulation. 2015;132(15):1435-1486. 